\title{
Research on a New Switchgear Maintenance Training System
}

\author{
Guo Zhuoli, Yang Guotai, Liang Jing \\ Shanxi Electric Power Company Training Center \\ Linfen, China \\ 1337967585@qq.com
}

\begin{abstract}
For the lack of effective means of Switchgear maintenance training, a new switchgear maintenance training system based on the power of large data was presented. This training system can provide typical switchgear maintenance projects, effectively improve safety awareness and maintenance skills of maintenance personnel, and improve the status quo of the existing switchgear maintenance, which basically relies on the seniors' help.
\end{abstract}

Keywords—big data; switchgear maintenance; training

\section{INTRODUCTION}

With the rapid development of power grid construction, scale and complexity of the grid system is also increasing, each information system has accumulated a great deal of status information data of power transmission equipments. This brings new problems and challenges to the operation and maintenance training. The traditional artificial teaching and training is expensive, the training effect is not high. Online education is now an emerging and advanced teaching management tools, it can make up for the shortcomings of the current traditional artificial teaching. Which effectively saves money and learning time but achieves optimal learning outcomes, and can effectively track and manage each student's learning outcomes. It can help power companies specialize in staff training, improve staff skills, promote human development, and to improve its core competitiveness by means of online education.

Especially the maintenance training of high-voltage, medium voltage switchgear, mainly achieve training through an apprentice chef way, this training method is not practical, and lacks of realism, the operator has no intuitive and concrete understanding on the various security risks that may occur during the operation, at the same time, no personal experience about serious consequences may arise due to a variety of misuse. Thus, when the operator enters the actual work, often causes accidents because of improper operation or poor safety awareness, which will cause great bodily injury and property damage.

Aiming the problems of switchgear maintenance training, a new switchgear maintenance training system based big data technology was developed in this paper, which was divided into three functional modules as online training, online testing and system management to achieve data sharing and knowledge sharing between training system and state evaluation system, and improve the level and efficiency of training.

\section{Power Big Data technologies}

The State Grid Corporation has build four data centers such as the structured data center, unstructured data management platform, massive historical quasi-real-time data management platform, grid geographic information service platform with the advance of construction. This makes grid accumulate a large number of data resources, grid data has been of considerable size from the amount and type, has a good data base, and initially achieves the integration and sharing of enterprise data resources. State Grid Corporation has entered the era of big data.

Based on the data processing and analysis of massive power transmission equipment state information, switchgear maintenance training system was set up for student to train routine switchgear maintenance operation, misuse and the corresponding consequences of operational errors and remedial measures to further improve the safety awareness of students and maintenance skills, bid farewell to the traditional mode that rely on master' experience, which will promote operation and maintenance training to standardization, efficient, network and visualization development.

\section{SWitchgear MaintenanCE TRAining System}

\section{A. System Framework}

Switchgear maintenance training platform based Big Data technology is a complete information platform, which includes typical project library, online training, online examinations, training resources, training needs research, training plans and reports, training objectives management, online communication, data statistical analysis and other multiple modules. Its network topology as shown in figure1. 


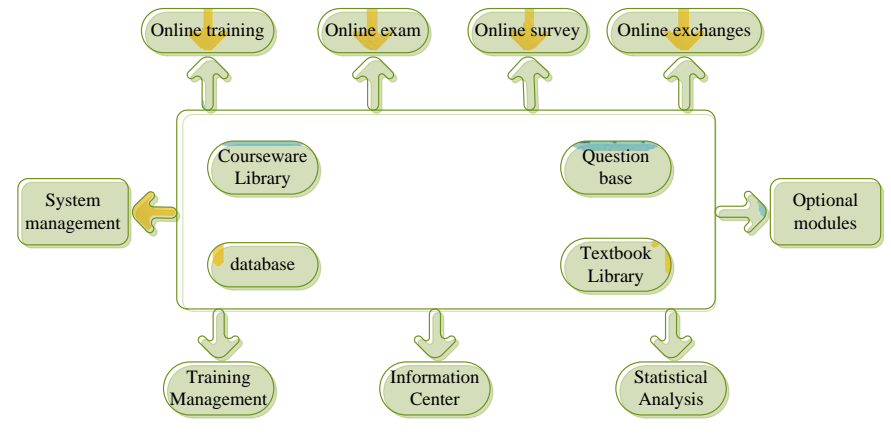

Fig. 1. System Network Topology

System is based on Hadoop distributed system infrastructure, the resulting information through monitoring and detection also has a multi-source heterogeneous characteristics due to the complexity of grid structure. Using Hadoop scheme to build a user base system is for the expansion of storage levels, MAPreduce approach is focused on the problem of parallel processing batch event, extracted data from on-line monitoring data, test data and live test data and other data sources and convert format, and import HBase for data storage. Fully draw the design ideas of multidimensional data model for the design of data storage model. Taking into account the needs of the application, multidimensional data was validly organized using row key, column key, column families, and other design flexibility in HBase. The query performance of HBase has been progress on the basis of REBMS mature technology. The unique feature of HBase that multi-version can co-exist can solve the problem of data patterns update.

The query like SQL provided by Hive is more suitable interface for data models, which makes data analysts can more easily design analysis algorithm. At the same time, the introduction of a distributed framework Mahout of machine learning and data mining makes creating intelligent applications more quickly and easily.

The system uses sqoop to extract data in a relational database, collects each system log using Flume, or supplements other ETL tools to obtain more comprehensive data. Data enters the data warehouse after cleaning, filtering and format conversion.

Data modeling module obtains user attributes, business use, behavioral preferences and other data from the data warehouse, builds user portrait and the user classification and clustering by the data model and collaboration rules. And then builds users view and sub business view.

\section{B. System Environment}

In accordance with the requirements of the State Grid Corporation, and following the relevant regulations and requirements of information construction, this paper chose the development platform that State Grid Corporation approved. Using the the thought of configuration and components to plan and design system to improve the stability, scalability of the system.
System is divided into application server, database server and streaming media server. Operating systems of application server and streaming media server requires Windows Server mainstream operating system, it is recommended to run under Windows 2003 Server / Windows 2008 Server operating system. System runs on IIS6.0 or above, .net4.0 version framework. Database support MS SQL Server, MySQL, Oracle and other mainstream database. Operating system of database server is determined depending on the specific type of database. The system is running in an open TCP / IP network environment, HTTP, HTTPS, SSL and other Internet protocols can be supported.

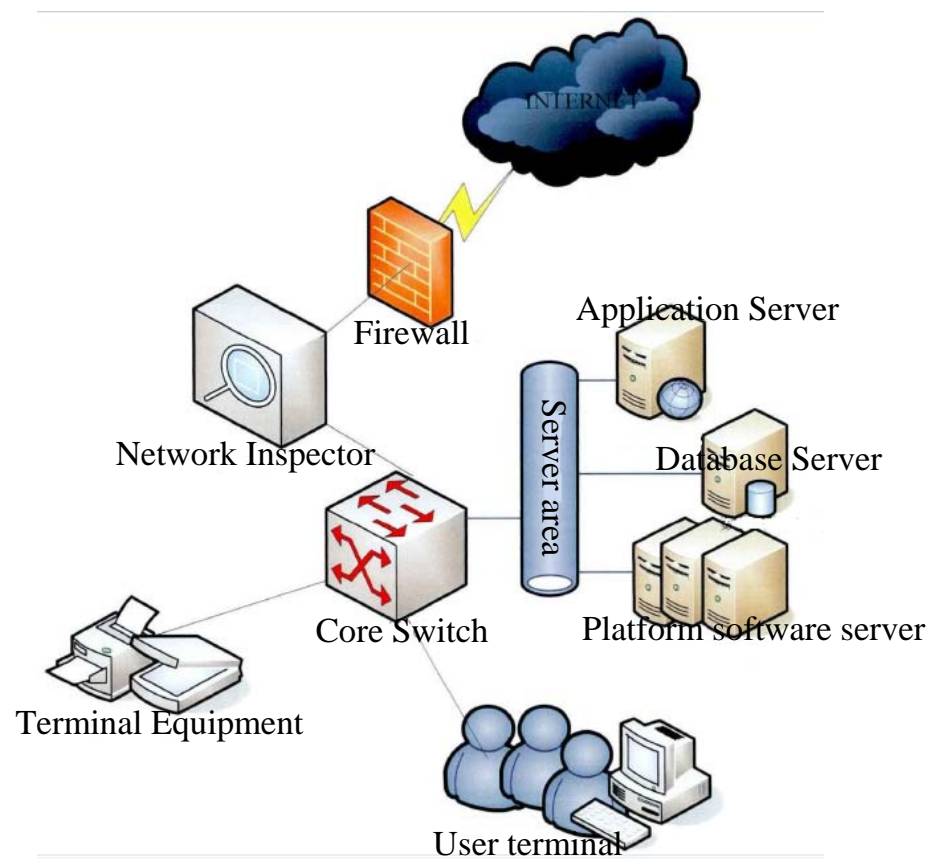

Fig. 2. System topology

\section{System functions}

Operation and maintenance comprehensive training platform is divided into three functional modules: online training, online exam and system management, while developing mobile client. The system supports the mobile client access, supports online questionnaires, video playback, supports for Android, IOS, Windows Phone three clients, and supports the display of gestures without refresh.

\section{1) Online Training}

Students can learn the basics by the way of instructional videos, case database, and other online self-study materials, but also can make students master the knowledge points more firmly through the relevant exercises and unit testing. The unit of courses is set up to manage the entire training process of certain models or trainees. Typical training projects are as follows: operated error numbers switchgear; SF6 gas pressure indicated an abnormal position, unchecked; pulled disconnectors with loads; mistakenly charged interval; furnished ground wire charged; unused qualified electrical inspection pen, not wear insulated gloves; not wear insulated gloves, the ground wire installed in the wrong order; the operation of running turning to maintenance is completed 
without hanging "DO NOT TURN ON, THE LINE IS WORKING" signage; power transmission from the rear end; closed disconnectors with ground; demolished ground without wearing insulated gloves, remove the grounding wire in error sequence.

\section{2) Online Axam}

Online exam can achieve examinations automatic, systematic and gridding, and realize papers management of the primary examination, intermediate examination and advanced examination by unified management of its examination papers and classification knowledge management.

Enterprise managers can easily organize large or small exams by online exam, test sites can be online remote operations, which may include certification exams, political learning test, new employees test, new business training and examinations.

\section{3) System Management}

System management module should be able to make educators and training managers manager roles and rights, and has parameter setting, profile, message notification and other functions.

\section{CONCLUSION}

For the problem that current Switchgear maintenance training lacks effective means, this paper presented a new switchgear maintenance training system based on the power big data technology. The system consists of online training, online testing and system management, which is able to offer typical switchgear maintenance projects training for trainees, improves students' safety awareness and maintenance skills, and it has an important applications.

\section{References}

[1] Huang yanhao, Yu zhihong, Shi dongyu, Zhou xiaoxin, "Study on the Application of Electric Power Big Data Technology in Power System Simulation," Proceedings of the CSEE, vol. 35 no.1, pp. 13-22, Iane 2015.

[2] Peng xiaosheng, Deng diyuan, Cheng shijie, Wen jinyu, Li zhaohui, Niu lin, "Key Technologies of Electric Power Big Data and Its Application Prospects in Smart Grid,” Proceedings of the CSEE, vol. 35 no.3, pp. 503-511, Feb. 2015.

[3] Zhang pei, Yang huafei, Xu yuanbin, "Power Big Data and Its Application Scenarios in Power Grid,” Proceedings of the CSEE, vol. 34 , pp.85-92, Nov. 2014.

[4] Feng ming, Wang sen, "10kV High Voltage Switchgear repair and maintenance considerations and Fault Case Analysis," Mechanical and Electrical Information, vol. 15, pp.52-53, 2014.

[5] Xie yanbin, Chen zheng, Zhou qian, "Network switchgear with safety risks Experience Training System based on the configuration of the wireless,” Electrotechnical Application, vol. S2, pp.502-509,2013.

[6] Zhao zirong, Design and implementation of $220 \mathrm{kV}$ substation simulation training and examination system. University of Electronic Science and Technology of China, 2012. 